\title{
Evaluating disease severity in idiopathic pulmonary fibrosis
}

\author{
Hasti Robbie ${ }^{1}$, Cécile Daccord ${ }^{2,3},{\text { Felix } \text { Chua }^{2} \text { and Anand Devaraj }}^{4}$ \\ Number 3 in the Series "Radiology" \\ Edited by Nicola Sverzellati and Sujal Desai
}

Affiliations: ${ }^{1}$ Radiology Dept, Royal Brompton and Harefield NHS Foundation Trust, London, UK. ${ }^{2}$ Interstitial Lung Disease Unit, Royal Brompton and Harefield NHS Foundation Trust, London, UK. ${ }^{3}$ Respiratory Medicine, University Hospital of Lausanne, Lausanne, Switzerland. ${ }^{4}$ Centre for Academic Radiology, Royal Brompton Hospital, London, UK.

Correspondence: Hasti Robbie, Radiology Dept, Royal Brompton and Harefield NHS Foundation Trust, Sydney Street, London, SW3 6NP, UK. E-mail: hasti.robbieanhs.net

@ERSpublications

Staging disease severity in IPF is challenging: a review of current available methods and future developments http://ow.ly/oBoJ30e8kVS

Cite this article as: Robbie H, Daccord C, Chua F, et al. Evaluating disease severity in idiopathic pulmonary fibrosis. Eur Respir Rev 2017; 26: 170051 [https://doi.org/10.1183/16000617.0051-2017].

ABSTRACT Accurate assessment of idiopathic pulmonary fibrosis (IPF) disease severity is integral to the care provided to patients with IPF. However, to date, there are no generally accepted or validated staging systems. There is an abundance of data on using information acquired from physiological, radiological and pathological parameters, in isolation or in combination, to assess disease severity in IPF. Recently, there has been interest in using serum biomarkers and computed tomography-derived quantitative lung fibrosis measures to stage disease severity in IPF. This review will focus on the suggested methods for staging IPF, at baseline and on serial assessment, their strengths and limitations, as well as future developments.

\section{Introduction}

Idiopathic pulmonary fibrosis (IPF), the most prevalent form of the idiopathic interstitial pneumonias (IIPs), is defined in international guidelines as "a chronic, progressively fibrosing IIP, occurring primarily in the lungs of older adults and associated with the histopathological and/or radiologic pattern of usual interstitial pneumonia (UIP)" [1]. Being behaviourally heterogeneous, it advances rapidly in some individuals to produce a stepwise loss of lung function culminating in early death, while progressing more insidiously with periods of apparent symptomatic and physiological stability in others (fig. 1). Overall, IPF is associated with poor prognosis and a median survival time of approximately 2-3 years, as reported by retrospective longitudinal studies dating from the late 1990s [2-6].

It is acknowledged that accurate assessment of IPF disease severity is central to the care that physicians provide to patients with IPF. Its importance spans the entire breadth of healthcare planning and resource provision, reimbursement of high-cost antifibrotic drugs and the design of clinical trials. One method to

Received: April 202017 | Accepted after revision: June 162017

Previous articles in this series: No. 1: Walsh SLF. Multidisciplinary evaluation of interstitial lung diseases: current insights. Eur Respir Rev 2017; 26: 170002. No. 2: Elicker BM, Kallianos KG, Henry TS. The role of high-resolution computed tomography in the follow-up of diffuse lung disease. Eur Respir Rev 2017; 26: 170008.

Conflict of interest: Disclosures can be found alongside this article at err.ersjournals.com

Provenance: Commissioned article, peer reviewed.

Copyright OERS 2017. ERR articles are open access and distributed under the terms of the Creative Commons Attribution Non-Commercial Licence 4.0. 

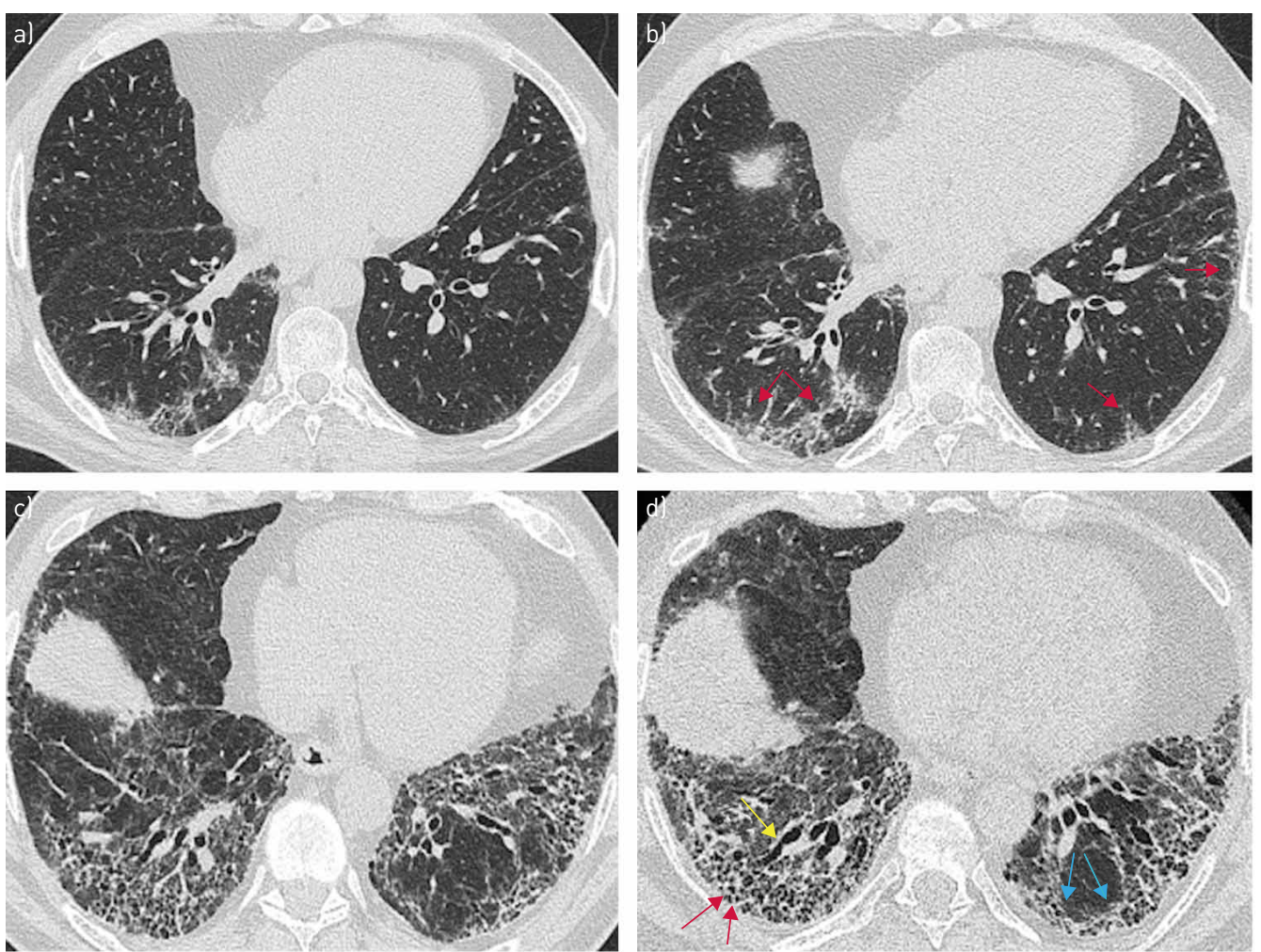

FIGURE 1 Axial computed tomography (CT) images a) at baseline and b) at a 12-month follow-up scan, in a patient with idiopathic pulmonary fibrosis (IPF). b) Note more progressive reticulation in the right lower lobe with new areas of subpleural reticulation in the left lower lobe (red arrows). Axial CT images in another patient with IPF cl at baseline and d) at a 12-month follow-up scan. d) Note the progressive honeycombing (red arrows), traction dilation of the airways (yellow arrow) and reticulation in both lobes (blue arrows).

assess IPF disease severity is by staging its extent using information acquired from physiological, radiological and pathological evaluation. As an example, based on the prevailing degree of pulmonary function impairment and/or the extent of radiological abnormalities, IPF severity has traditionally been described using imprecise terms such as "mild", "moderate", "severe", "early" or "advanced" disease. Not surprisingly, this approach has not gained widespread acceptance or been co-opted into routine clinical practice. Its true prognostic value remains unclear [1].

Data potentially exist to permit more considered judgements to be made on the assessment of disease severity using more precise variables. Indeed an increasing body of knowledge is emerging to shed light on the relationship between selected clinical, physiological, imaging and pathological factors and mortality in IPF [1]. Attempts have been made to integrate these determinants to refine the prognostication process [7-9]. The potential utility of serum biomarkers as additional indicators of IPF disease severity is gaining wider attention; the integration of biological mediator measurements or patterns of expression into diagnostic and prognostic systems will undoubtedly be a prime focus of future IPF research [10-15].

The current absence of a generally adopted or validated tool for staging IPF is testament to the uncertainty surrounding the optimal manner to approach this issue. This review will focus on the current means of staging IPF severity at baseline and on serial assessment, their strengths and limitations, as well as future developments.

Why stage patients with IPF?

Advocates of IPF staging cite a number of reasons in its support, among them a desire to improve the quality of information that can be communicated to patients and their carers, as well as to help identify clinical thresholds for initiating treatment, particularly in healthcare systems where such treatment is tightly regulated. Additionally, accurate staging algorithms that can sensitively distinguish early progressive from chronic stable disease may help in refining ongoing therapeutic decisions. A reliable method for highlighting patients as they progress to more advanced stages of disease is also desirable, while enabling the earlier identification of complications such as pulmonary hypertension and/or right heart failure, or prompting a timely referral for transplant assessment or specialist end-of-life care. From a research 
standpoint, therapeutic trials require sensitive markers that evaluate the change in severity of disease over time. Furthermore, cases of mild to moderate severity IPF identified by a standardised staging protocol may be more efficiently targeted for inclusion in observational or interventional trials [16].

\section{Clinical, physiological and functional markers of disease severity}

A number of clinical, physiological and functional variables, captured at baseline and longitudinally, have been associated with an increased risk of mortality in IPF. Nevertheless, it is still not possible to confidently detect IPF at an early stage, evaluate its severity in a clinically consistent manner, predict the likelihood of response to treatment, or foretell its subsequent disease behaviour sensitively using a single marker. The heterogeneity of phase 3 IPF trial characteristics illustrates the lack of a standardised approach to study design as well as disagreement about optimal primary and secondary end-points. A recent consensus effort including contributions from interstitial lung disease (ILD) experts and patients proposed the adoption of a core set of domains and outcome measures in future clinical trials of connective tissue disease-associated ILD (CTD-ILD) and IPF, but has yet to be put to the test $[17,18]$.

\section{Clinical markers of disease severity in IPF}

Dyspnoea and cough are the most common symptoms of IPF and can impact significantly on the daily activities and health-related quality of life (HRQoL) of affected individuals. However, symptom severity per se is notoriously difficult to quantify. The assessment of dyspnoea using the University of California San Diego Shortness of Breath Questionnaire (UCSD-SBQ) has been used as a secondary end-point in a phase 3 trial [19]. Meanwhile, the Medical Research Council (MRC) breathlessness scale has been used to aid the stratification of IPF disease severity [20]. When assessed longitudinally, the latter has also been shown to predict clinical progression [21]. Although inexpensive and relatively user-friendly, most dyspnoea assessment instruments have not been developed specifically for ILD cases such as IPF [18].

Cough has also been associated with disease progression in IPF [22]. Cough scales, questionnaires, challenge tests and monitors are among the methods used to assess cough severity in IPF and its impact on HRQoL [23]. The Leicester Cough Questionnaire (LCQ) has been shown to be sensitive to serial change and is therefore considered an appropriate outcome tool in IPF trials [17, 24].

\section{Physiological markers of disease severity in IPF}

Forced vital capacity (FVC) and, to a lesser extent, diffusing capacity of the lung for carbon monoxide (DLCO) are the most commonly used lung function parameters for denoting IPF severity, progression and response to treatment. Longitudinal decline in FVC appears to better predict disease progression and mortality than baseline values $[25,26]$. Consequently, this parameter has become the preferred clinical end-point in treatment trials. An absolute decline in FVC of $\geqslant 10 \%$ or in DLCO of $\geqslant 15 \%$ over 6 months has been regarded as clinically important and is frequently used to describe "significant" disease progression [27]. RicheLDi et al. [28] have proposed using the relative rather than absolute decline in $10 \%$ of the FVC to maximise the chance of detecting disease progression with similar prognostic accuracy.

Existing data also suggest that a "marginal" (5-10\%) decline in percent-predicted FVC at 6 months is associated with increased mortality in IPF and that the minimal clinically important difference (MCID) for this parameter is estimated to be between $2 \%$ and $6 \%$ of the baseline measurement $[26,29]$. The assessment of the slope of change in FVC using serial measurements reduces the inherent variability of FVC measurement compared with the difference in FVC measured at two time points. It has been proposed that daily home monitoring of FVC with a portable spirometer can provide a more sensitive and earlier predictor of disease behaviour and outcome than periodic hospital-based measurement of the same marker [30]. In this report, the rate of decline in FVC on daily home spirometry was shown to be highly predictive of mortality, from as early as 3 months. These results suggest that daily FVC recording might have value as an efficacy end-point, where its use could shorten the duration of early-phase clinical trials. In the clinical arena, trends detected by daily FVC monitoring might also alert the patient and physician earlier to disease progression or the onset of an acute exacerbation episode.

Grouped analyses of patients with FVC $\geqslant 50 \%$ predicted in the ASCEND and INPULSIS trials showed the utility of FVC as a marker of IPF progression and its sensitivity to treatment-associated change [19, 31]. However, many patients presenting to clinical practice with $\mathrm{FVC}<50 \%$ predicted and/or significantly reduced DLCO consequent upon severe fibrotic disease, coexisting chronic obstructive pulmonary disease or pulmonary hypertension are excluded from trials. Trial findings may not entirely apply to most patients in the real world because their disease severity falls within a much broader range of physiological impairment than in trial settings. What has been directly relevant to clinical practice is the demonstration in INPULSIS-ON, the open-label extension of the INPULSIS trials of nintedanib in IPF, that FVC decline 
is comparable over a 48-week period of treatment between individuals whose FVC lay on either side of the $50 \%$ predicted mark [32].

The STEP-IPF study of sildenafil in advanced IPF enrolled patients with DLCO of $<35 \%$ of predicted [33]. The mortality rate of $13 \%$ at 28 weeks in this cohort of subjects with severe disease contrasts with the 2-year $13.7 \%$ fatality rate revealed by the pooled analysis of the placebo arms of the INSPIRE and CAPACITY trials that included patients with "mild-to-moderate" IPF [34]. These results demonstrate the correlation between risk of death and IPF disease severity. In reality, however, it is precisely because recent trials have been beset by a low mortality rate that they have had to rely on pooled data analyses to highlight the potential effect of antifibrotic therapy in modulating the risk of disease-related death.

Similarly, pooled and meta-analyses of clinical trials of pirfenidone have shown that the relative risk of death in IPF is reduced over 120 weeks of treatment [35]. It seems that continued treatment with pirfenidone in those whose disease has progressed, defined as $\geqslant 10 \%$ absolute decline in FVC during the first 6 months of treatment, can still be beneficial as reflected in a lower risk of subsequent FVC decline or death [36].

Post hoc subgroup analyses of both pirfenidone and nintedanib trials have shown that patients with so-called "preserved lung volume" (FVC $>90 \%$ predicted in the nintedanib subgroup and FVC $\geqslant 80 \%$ in the pirfenidone subgroup) have a rate of FVC decline comparable to those with moderate or severe disease and that they also benefit from antifibrotic therapy to the same extent as patients with more contracted lung volume $[37,38]$. Hence, once a confident diagnosis of IPF has been made, it could be reasonably questioned if the precise categorisation of disease into very mild, mild or moderate groups is necessary for eligibility for antifibrotic therapy. Crucially, these observations highlight the importance of securing a timely diagnosis of IPF, but this process suffers from the absence of clinical tools to distinguish early IPF from insignificant interstitial lung abnormalities that are increasingly encountered in chronic smokers. Such changes, whether or not representing smoking-related ILD, often remain subclinical and, for the most part, do not progress over time $[39,40]$.

Severe IPF appears to be also associated with a higher risk of acute exacerbations [31]. It is thus possible to postulate that by slowing the rate of progression, quantified as a delay in the loss of FVC, antifibrotic strategies might delay the time to exacerbation. It may now be difficult or impossible to study this premise, as it would be unethical to withhold antifibrotic treatment to patients with a certain severity of IPF while prospectively monitoring them for the development of acutely deteriorating disease.

Data regarding change in percentage-predicted DLCO as a marker for predicting survival in IPF are inconclusive. A retrospective analysis of treatment with interferon- $\gamma 1 \mathrm{~b}$ failed to identify a threshold of DLCO change that predicted mortality, although an earlier study showed that trends in DLCO levels might provide important information for determining mortality [41, 42]. It has latterly been proposed that baseline DLCO outperforms baseline FVC as a prognostic factor in IPF [25]. An analysis of a UK prospective IPF cohort has similarly suggested that DLCO is better than FVC as a predictor of mortality, especially in patients with specific risk factors that portend a worse prognosis, such as concomitant emphysema and pulmonary hypertension [43]. In a study of patients with IIP, both baseline and 6-month decline in KCO (diffusing capacity adjusted for functioning alveolar volume) were associated with increased early and overall mortality [44]. These results suggest that FVC may not be the sole or even the best predictor of fibrotic disease progression or mortality in real-world clinical cohorts when serial physiological changes are assessed.

Spirometric parameters have certain limitations when used longitudinally. It is difficult to ascertain whether longitudinal change is due to test variability or reflects a true change in lung function [45]. FVC can be poorly sensitive to change and marginal changes of 5-10\% are difficult to interpret in an individual [46]. Measurements of DLCO and KCO are less widely available and more difficult to perform in individuals with severe disease. They are also subject to greater intra-individual and inter-laboratory variability than FVC and are sensitively affected by comorbid conditions such as emphysema or pulmonary hypertension.

\section{Functional markers of disease severity in IPF}

Baseline and change at 6 months of the 6 -min walk distance (6MWD) have been reported as independent predictors of mortality in IPF [47]. The MCID for 6MWD has been estimated to be between $21.7 \mathrm{~m}$ and $37 \mathrm{~m}$ in a cohort of patients with IPF allocated to the placebo arms of the CAPACITY trials [48]. This, as well as findings from an earlier interferon $\gamma$-1b trial, confirm the utility and validity of the 6MWD as a measure of disease status and end-point for IPF clinical trials [49]. Desaturation on 6MWT has also been associated with mortality in IPF, but measurement of oxygen desaturation is hampered by poor reproducibility $[50,51]$. Interestingly, abnormal heart rate recovery after $6 \mathrm{MWT}$ or the difference between 
the heart rate at the end and at $1 \mathrm{~min}$ after completion of the test has shown to be an independent predictor of mortality and pulmonary hypertension in patients with IPF [52, 53].

In conclusion, although it is increasingly accepted that a number of factors such as interval change in FVC, dyspnoea and 6MWD can individually or collectively correlate with a particular severity of IPF, this does not equate to being reliable predictors of longitudinal disease progression. Indeed, when incorporated within clinical prediction models, they perform better at predicting binary outcomes like respiratory hospitalisation and death than intrinsic disease progression [54]. This highlights the need for the development and validation of tools that have the potential to predict disease behaviour and risk of progression which would be useful in clinical decision-making and designing future clinical trials.

\section{Radiological markers of disease severity Semi-quantitative computed tomography}

The most pertinent computed tomography (CT) patterns of fibrotic ILD are reticulation, traction bronchiectasis, honeycombing and ground-glass opacification, which have specific and broadly agreed definitions [55].

Visual assessment of disease extent can be reported with poorly defined terms like mild, moderate or severe, or can be reported as an estimate of the percentage of the lung affected to the nearest $5 \%, 10 \%$ or $25 \%$. This mental mapping of the disease extent may be easier to do on a single CT section than from a three-dimensional display of the lung [46]. To address this issue, a hybrid method of dividing the lung into three or five sections based on the corresponding mediastinal structures has been widely used $[9,56,57]$.

Several studies have investigated the relationship between extent of fibrosis on CT and outcomes at a single point in time. Baseline patterns, in particular honeycombing, the hallmark of UIP/IPF, have also been widely investigated as a marker of outcome (with some disparity in study results) [58-63]. LYNCH et al. [58], using non-volumetric CT, found that the overall extent of fibrosis, defined as the extent of reticulation and honeycombing on CT, was an independent predictor of mortality in IPF. Similarly, studies by SHIN et al. [64] and EDEY et al. [61] found that the overall extent of fibrosis indicated a poor prognosis in populations that contained not only patients with IPF but other fibrotic IIPs. The results from these studies indicate that increasing disease extent on CT is undeniably related to adverse prognosis in IPF. However, clinically useful staging systems based on imaging (such as those used in the setting of cancer staging) traditionally require absolute cut-offs that demarcate different prognostic groups. In this regard, there is a paucity of research in IPF. In one study by ROMEI et al. [65] it was shown that baseline honeycombing $>25 \%$, fibrosis score $>30 \%$ and traction bronchiectasis in all four lobes were indicators of poor outcome.

The relationship between CT patterns, and not just disease extent, has also been studied in IPF. In a study of 98 patients with histologically confirmed diagnosis of IPF, SumiKawa et al. [66] categorised patients into three groups: 1) definite UIP; 2) consistent with UIP; and 3) suggestive of an alternative diagnosis. No significant difference in prognosis between the different groups was found, but prognosis was influenced by traction bronchiectasis and overall fibrosis scores. More recently, there are data suggesting that many cases with a possible UIP pattern (i.e. no honeycombing on baseline high-resolution CT (HRCT)) follow a comparable trajectory to those with a definite UIP pattern (i.e. honeycombing on baseline HRCT). A post hoc subgroup analysis from the INPULSIS trials suggested that patients with a diagnosis of IPF based on possible UIP pattern on HRCT showed similar rate of decline in FVC as patients with definite UIP pattern based on the presence of honeycombing on HRCT or histological diagnosis of UIP [63].

There are fewer studies looking at serial CT to monitor disease progression in IPF using a visual assessment of disease extent. LeE et al. [67] studied 154 patients who had fibrotic IIP with little honeycombing (defined as $<5 \%$ ). The authors found that an increase in honeycombing and reticulation on serial CT was associated with poorer outcomes. However, on multivariate analysis, the extent of fibrosis on the baseline scan remained the only predictor of mortality. By contrast, in another study, YAMAUCHI and colleagues [62] demonstrated that the development of honeycombing on HRCT during follow-up in 30 patients with classical or possible IPF did not affect prognosis. Hwang et al. [68] also looked at the prognostic value of $\mathrm{CT}$ at baseline and on sequential evaluation in a heterogeneous group of fibrosing IIPs. The investigators found both the extent of honeycombing at baseline and its progression on serial imaging to be independent determinants of mortality. A study by ODA et al. [69] was particularly notable as it studied patients over a comparatively shorter time frame. The authors found that increasing visual fibrosis score on a 6-month follow-up CT was a significant predictor of poorer outcomes, even in a subgroup of patients with no decline in FVC. The results from these studies illustrate the lack of clear consensus about both the utility of subjectively comparing fibrosis extent on serial CT, as well as its significance. In part, this may reflect the difficulties in visually comparing overall fibrosis extent over the entirety of a thoracic 
CT. Nevertheless, there are also instances where disease progression on CT is unequivocal, which may confirm suspicions of clinical deterioration.

\section{Quantitative computed tomography}

Quantitative CT is a relatively new and promising domain in the evaluation of disease extent in ILD. Methods vary from simple histogram analysis to more complex forms of textural based analysis and machine learning. In the former, histogram-based measurements such as mean lung attenuation and amount of skewness and kurtosis from CT images have been found to correlate with physiological measures of disease severity [46, 70-72].

Automated textural analysis typically requires, as an initial step, trained observers labelling regions of interest in the lung according to a specific pattern (e.g. normal, honeycombing, etc.). Textural characteristics of these regions are then extracted. A machine-learning algorithm is then used to develop a predictive model for specific CT patterns [46]. MALDONADO et al. [73] used a novel computer-aided system called CALIPER to quantify fibrosis in IPF based on unique HRCT texture patterns for ground-glass opacification, honeycombing and reticulation. The authors evaluated changes in those HRCT-derived patterns between two time points (3-15 months apart) and showed that changes in reticulation and total fibrosis were predictive of survival. Recently, ЈАСОВ et al. [74] compared quantitatively assessed disease extent on HRCT with visual scores in IPF. When the two techniques were validated against lung function test parameters, CALIPER was superior to the traditional visual scores. The investigators also found that a novel parameter called pulmonary vessel volume (PVV) had stronger correlations with lung function tests, indicating that it may have potential in assessing disease severity in IPF. The same group demonstrated that CALIPER-derived honeycombing and PVV were more accurate than traditional fibrosis scores in predicting mortality, independent of physiological indices [75]. While quantitative disease assessment in lung fibrosis has been around for some years, its application in monitoring disease in therapeutic trials has recently gained interest. One notable recent study in this field by SALISBURY et al. [76] involved textural analysis of a cohort of IPF patients enrolled in a clinical therapeutic trial using a novel adaptive multiple features method (AMFM) at two time points. The investigators observed that AMFM-measured fibrosis was independently related to the risk of disease progression as judged by a number of clinical and physiological measures, including the composite gender-age-physiology (GAP) score (a score accounting concurrently for patient age, gender, FVC and DLCO, where a higher score is associated with increased risk of mortality) and smoking history. These studies suggest that quantitative lung fibrosis assessment has the potential to emerge as a powerful biomarker of IPF severity and disease progression, but future work is required to validate its role.

\section{Strengths and limitations of CT in staging of IPF}

There are several potential advantages to using CT for staging patients in IPF. The acquisition of images is fast and routinely available, allowing adequate assessment of stable as well as very ill patients. Coexisting conditions and complications such as emphysema, pulmonary hypertension and acute exacerbations can be effectively and simultaneously assessed on CT. Moreover, CT findings correlate accurately with functional impairment [9]. One of the main limitations with CT is the lack of standardised acquisition protocols. For example, non-contiguous CT is still routinely performed at some centres, and many of the studies that report on the role of CT in prognostication in IPF used interspaced CT. Because volumetric CT captures images of the entire thorax, it is assumed that it can produce more accurate information about disease extent, in particular with respect to serial change. However, the extent to which this is the case is not truly known. The state of lung inflation at the time of image acquisition also impacts lung density, so standardised breathing instructions at the time of image acquisition are essential for congruous results. Variation in lung density is at its lowest at full inspiration, when consistent results are thus more likely to be achievable [46].

The interobserver agreement for CT patterns, and in particular honeycombing, remains suboptimal due to concomitant conditions such as emphysema or traction bronchiectasis that mimic honeycombing [7, 67, 77]. WATADANI et al. [77] used a five-point scale to assess agreement for honeycombing on CT amongst 43 observers in 80 patients with IPF. In almost a third of the cases, there was disagreement on the identification of honeycombing due to the conditions resembling honeycombing on CT. Interobserver variation in assessing progression of disease on CT has been less widely studied.

Visual scoring of the disease extent on CT can be time-consuming, and its applicability in clinical practice is limited. There is no standardised method of visual scoring, and its value in serial assessment is not fully validated [46]. Furthermore, most data are derived from retrospective studies with varying follow-up intervals between serial CT scans. Additionally, retrospective studies that include clinically indicated CT scans may be biased towards including patients with disease deterioration. However, retrospective studies 
using real-world clinical data have the advantage of incorporating patients with varying extents of disease severity.

Quantitative CT has the potential to diminish but not entirely eliminate interobserver disagreement, considering that the software remains dependent on training by expert radiologists, who are subject to interobserver variability. In addition, to date, it has primarily focused on evaluating the extent of parenchymal abnormalities as a surrogate for fibrosis severity, with less emphasis on other variables such as lung volumes $[73,74]$ or the degree of traction bronchiectasis. The issue of CT protocol standardisation is nevertheless equally important, if not more so, for automated evaluation. Furthermore, issues such as cost, reproducibility and validity need to be addressed before such computer-aided systems can routinely be used in clinical practice or clinical trials.

\section{Positron emission tomography CT}

There is limited evidence that ${ }^{18} \mathrm{~F}-\mathrm{FDG}$ positron emission tomography (PET) can be used in the assessment of IPF or other forms of IIPs [78-82]. GRoves et al. [79] investigated the relationship between mean maximal standard uptake value $\left(\mathrm{SUV}_{\max }\right)$ values and lung function parameters (FVC and DLCO) in a cohort of 36 patients with diffuse lung disease (18 with IPF). They found moderate correlations between pulmonary ${ }^{18} \mathrm{~F}-\mathrm{FDG}$ uptake and lung function parameters. The investigators also found that glucose metabolism was higher in areas with reticulation/honeycombing than in regions with ground-glass opacification $\left(\mathrm{SUV}_{\max }=3.0+/-1.0\right.$ and $2.1+/-0.4$ respectively, $\left.\mathrm{p}=0.007\right)$. In a study of 50 patients with various IIPs comprising IPF, nonspecific interstitial pneumonia (NSIP) and organising pneumonia, UMEDA and colleagues [81] investigated the value of dual-point ${ }^{18} \mathrm{~F}-\mathrm{FDG}$ PET CT (performed $1 \mathrm{~h}$ and $3 \mathrm{~h}$ post ${ }^{18}$ F-FDG injection) in the diagnosis and prognostication of IPF. The authors used SUV values at the two points (early SUV at $1 \mathrm{~h}$ and delayed SUV at $3 \mathrm{~h}$ ) to calculate the retention index of SUV (RI-SUV) and then compared the results with serial lung function tests performed at 3 and 12 month intervals from the PET CT. They found that an early SUV cut-off value of $\geqslant 1.5$ had a high sensitivity and specificity (90.9 and 94.3, respectively) in distinguishing between organising pneumonia and IPF/NSIP. The RI-SUV was significantly higher in patients with IPF or NSIP that had a decline in lung function after 1 year follow-up. The same investigators also reported that positive RI-SUV $(\geqslant 0 \%)$ predicted a decline in lung function and mortality [82]. In this cohort, patients with positive RI-SUV tended to have more fibroblastic foci, and the authors concluded that RI-SUV may be associated with the degree of fibroblast activity in patients with IPF.

\section{Magnetic resonance imagining}

There are a handful of studies investigating various magnetic resonance imaging (MRI) sequences such as spin echo, inversion recovery or dynamic post-contrast T1-weighted multishot turbo field-echo sequences in IPF [83-86]. The principle for the majority of these studies is based on the assumption that, in contrast to normal lung tissue, fibrotic lung tissue demonstrates significant contrast enhancement on late-enhanced MRI sequences, which is a well-established fact in the assessment of myocardial fibrosis on cardiac MRI. In a pilot study of 10 patients with IPF and 10 healthy controls, MirsadraEe and colleagues studied T1 mapping at 3T MRI [87]. In this study, a modified look-locker inversion recovery (MOLLI) sequence was used during a 15-20 s breathhold on a single pre-selected slice based on HRCT findings. MOLLI sequences were acquired prior and at 15, 25, 30 and $35 \mathrm{~min}$ post gadolinium administration. The authors found that the fibrotic lung had the highest pre-contrast $\mathrm{T} 1$ and at $10 \mathrm{~min}$ post gadolinium administration, the control lung had a significantly shorter $\mathrm{T} 1$ compared to both fibrotic and morphologically normal lung in IPF. At $20 \mathrm{~min}$, T1 for fibrotic lung continued to decrease, but T1 for morphologically normal lung in IPF did not significantly change after $10 \mathrm{~min}$. This demonstrates that fibrotic lung shows delayed enhancement compared with morphologically normal lung. T1 mapping at 3T MRI in IPF can potentially differentiate between normal lung and fibrotic tissue and may be valuable in detecting early fibrosis and assessing disease activity if validated in larger cohorts.

Nevertheless, the use of MRI in IPF remains limited. This is because of a number of limiting factors associated with MRI in the lungs. The thick slices routinely used in MRI diminish the precise anatomical evaluation of interstitial changes. Furthermore, issues such as low proton density, fast signal decay due to artefacts/air tissue interfaces and prolonged acquisition times in patients with severe dyspnoea can reduce image quality and result in reduced diagnostic accuracy [84]. The majority of studies investigating the role of MRI in IPF are limited by small-sized cohorts and lack of validation. The other important issue is that the possible impact of various patterns (definite UIP, possible UIP and inconsistent with UIP) on MRI signal characteristics has not been investigated previously.

\section{Composite measures of disease severity}

Clinical prediction models in IPF may be enhanced by the use of composite indices to increase the accuracy and sensitivity of severity stratification and prognostication [18]. The ideal non-invasive 
TABLE 1 Comparison of mortality risk scoring systems in idiopathic pulmonary fibrosis

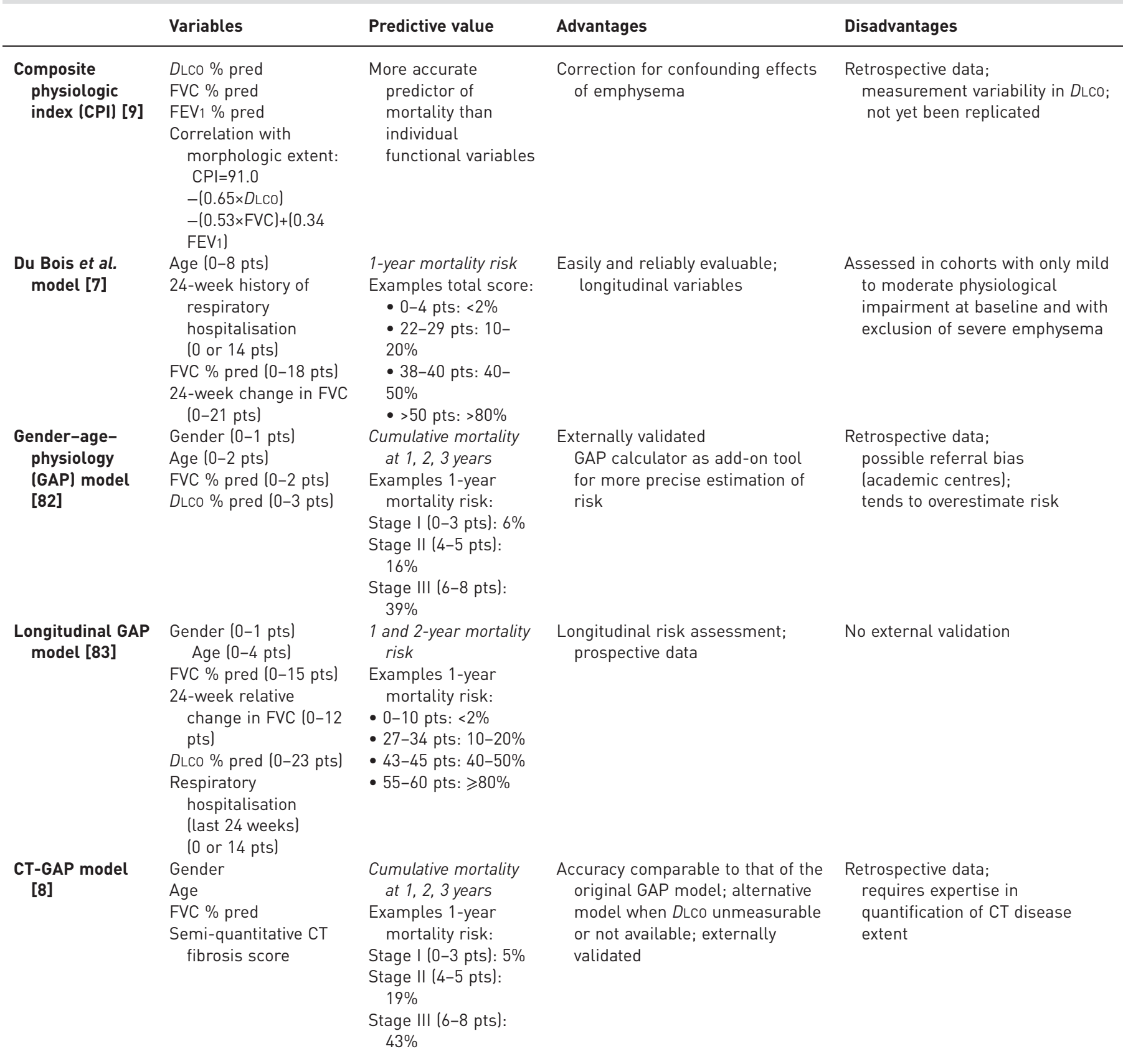

DLCo: diffusing capacity of carbon monoxide; FVC: forced vital capacity; FEV1: forced expiratory volume in $1 \mathrm{~s}$; CT: computed tomography; \% pred: \% predicted; pts: points. Reproduced and modified from [89] with permission from the publisher.

composite measure should incorporate different disease domains that have individually been shown to be reproducible, discriminant and sensitive to change over time. Several multidimensional risk prediction models integrating various clinical, physiological and radiological variables have been validated in IPF [79, 88-90] (table 1). These composite staging systems have been demonstrated to be better at predicting baseline and longitudinal mortality risk than their individual components. For example, the composite physiologic index (CPI) (table 1) has been shown to correlate better with disease extent on HRCT than individual functional variables, and allows correction of the confounding effects of emphysema on lung function parameters [9]. Another system called the GAP staging system, which incorporates gender, age, FVC and DLCO, has been shown to be predictive of death or lung transplantation but not of the future rate of decline in pulmonary function [91]. This model performs equally well when substituting DLCO for visually assessed CT fibrosis extent (CT-GAP score) [8]. 
A strategy to improve the sensitivity of future composite measures would be to integrate independent predictors of disease progression, such as serial change in FVC, with decline in 6MWD and/or serial change in HRCT fibrosis score $[18,92]$. Recent advances in our understanding of the complex pathogenesis of IPF have increased endeavours aimed at identifying and validating specific biological determinants that hold greater promise for diagnostic and prognostic applicability. Intuitively, the next step in the development of composite measures that can more accurately assess IPF severity and progression would probably include combinations of biomarkers.

\section{Histopathological staging in IPF}

A histologic pattern of UIP is associated with worse prognosis [93] and is characterised by the presence of fibroblastic foci in the interface between the fibrotic and the relatively spared regions of the pulmonary lobule [94]. Nevertheless, survival does not differ in patients with concordant UIP (UIP in all lobes) versus discordant UIP (UIP in at least one lobe) [93]. Although this indicates that the mere presence of UIP on pathology is significantly prognostic, there is also evidence that the number of fibroblastic foci on lung biopsy is related to outcomes [4,95]. Recently, WALSH and colleagues [96] investigated the relationship between fibroblastic foci and individual CT patterns of fibrosis. They found that severity of traction bronchiectasis correlated most strongly with fibroblastic foci profusion on histopathological analysis, providing an insight into the pathological processes behind traction bronchiectasis.

Pathological staging does have limitations. Surgical biopsy is required for histopathological diagnosis, but this only applies to perhaps $15 \%$ of IPF cases because of the severity of the disease at the time of diagnosis and the risks of biopsy [97]. Moreover, there can be suboptimal agreement among pathologists regarding the type of IIP. NichOLSON et al. [98] showed that a 100\% confidence level for a single pathologically made diagnosis was only reached in $39 \%$ of cases. The agreement amongst pathologists was poor for NSIP $(\kappa=0.32)$ and fair for UIP $(\kappa=0.49)$.

\section{Summary}

In conclusion, the accurate evaluation of disease severity in IPF and predicting disease progression is challenging and somewhat limited by a lack of standardised and validated measures. Pulmonary function parameters at a single time point do not reliably predict disease behaviour and, despite multiple attempts in the last 20 years or so, HRCT-quantified disease extent on sequential imaging has not been established as a reliable marker of disease progression. With advances in our understanding of disease behaviour in IPF, there are novel tools such as biological mediators or quantitative CT-derived lung fibrosis measures that can lead to the development of more robust staging systems, either in isolation or in combination. Future studies focusing on this area of research are certainly required to establish such systems.

\section{References}

1 Raghu G, Collard HR, Egan JJ, et al. An official ATS/ERS/JRS/ALAT statement: idiopathic pulmonary fibrosis: evidence-based guidelines for diagnosis and management. Am J Respir Crit Care Med 2011; 183: 788-824.

2 Bjoraker JA, Ryu JH, Edwin MK, et al. Prognostic significance of histopathologic subsets in idiopathic pulmonary fibrosis. Am J Respir Crit Care Med 1998; 157: 199-203.

3 Rudd RM, Prescott RJ, Chalmers JC, et al. British Thoracic Society Study on cryptogenic fibrosing alveolitis: response to treatment and survival. Thorax 2007; 62: 62-66.

4 King TE, Schwarz MI, Brown K, et al. Idiopathic pulmonary fibrosis: relationship between histopathologic features and mortality. Am J Respir Crit Care Med 2001; 164: 1025-1032.

5 Nicholson AG, Colby TV, du Bois RM, et al. The prognostic significance of the histologic pattern of interstitial pneumonia in patients presenting with the clinical entity of cryptogenic fibrosing alveolitis. Am J Respir Crit Care Med 2000; 162: 2213-2217.

6 Flaherty K, Toews G, Travis W, et al. Clinical significance of histological classification of idiopathic interstitial pneumonia. Eur Respir J 2002; 19: 275-283.

7 Du Bois RM, Weycker D, Albera C, et al. Ascertainment of individual risk of mortality for patients with idiopathic pulmonary fibrosis. Am J Respir Crit Care Med 2011; 184: 459-466.

8 Ley B, Elicker BM, Hartman TE, et al. Idiopathic pulmonary fibrosis: CT and risk of death. Radiology 2014; 273: $570-579$.

9 Wells AU, Desai SR, Rubens MB, et al. Idiopathic pulmonary fibrosis: a composite physiological index derived from disease extent observed by computed tomography. Am J Respir Crit Care Med 2003; 167: 962-969.

10 Satoh H, Kurishima K, Ishikawa H, et al. Increased levels of KL-6 and subsequent mortality in patients with interstitial lung diseases. J Intern Med 2006; 260: 429-434.

11 Kinder BW, Brown KK, McCormack FX, et al. Serum surfactant protein-A is a strong predictor of early mortality in idiopathic pulmonary fibrosis. Chest 2009; 135: 1557-1563.

12 Prasse A, Probst C, Bargagli E, et al. Serum CC-chemokine ligand 18 concentration predicts outcome in idiopathic pulmonary fibrosis. Am J Respir Crit Care Med 2009; 179: 717-723.

13 Richards TJ, Kaminski N, Baribaud F, et al. Peripheral blood proteins predict mortality in idiopathic pulmonary fibrosis. Am J Respir Crit Care Med 2012; 185: 67-76.

14 Sokai A, Handa T, Tanizawa K, et al. Matrix metalloproteinase-10: a novel biomarker for idiopathic pulmonary fibrosis. Respir Res 2015; 16: 120. 
15 Jenkins RG, Simpson JK, Saini G, et al. Longitudinal change in collagen degradation biomarkers in idiopathic pulmonary fibrosis: an analysis from the prospective, multicentre PROFILE study. Lancet Respir Med 2015; 3: $462-472$.

16 Azuma A, Taguchi Y, Ogura T, et al. Exploratory analysis of a phase III trial of pirfenidone identifies a subpopulation of patients with idiopathic pulmonary fibrosis as benefiting from treatment. Respir Res 2011; 12 : 143.

17 Saketkoo LA, Mittoo S, Huscher D, et al. Connective tissue disease related interstitial lung diseases and idiopathic pulmonary fibrosis: provisional core sets of domains and instruments for use in clinical trials. Thorax 2013; 69: $436-444$.

18 Lammi MR, Baughman RP, Birring SS, et al. Outcome measures for clinical trials in interstitial lung diseases. Curr Respir Med Rev 2015; 11: 163-174.

19 King TE, Bradford WZ, Castro-Bernardini S, et al. A phase 3 trial of pirfenidone in patients with idiopathic pulmonary fibrosis. N Engl J Med 2014; 370: 2083-2092.

20 Papiris SA, Daniil ZD, Malagari K, et al. The Medical Research Council dyspnea scale in the estimation of disease severity in idiopathic pulmonary fibrosis. Respir Med 2005; 99: 755-761.

21 Khadawardi H, Mura M. A simple dyspnoea scale as part of the assessment to predict outcome across chronic interstitial lung disease. Respirology 2016; 22: 501-507.

22 Ryerson CJ, Abbritti M, Ley B, et al. Cough predicts prognosis in idiopathic pulmonary fibrosis. Respirology 2011; 16: 969-975.

23 Van Manen MJG, Birring SS, Vancheri C, et al. Cough in idiopathic pulmonary fibrosis. Eur Respir Rev 2016; 25: 278-286.

24 Key AL, Holt K, Hamilton A, et al. Objective cough frequency in idiopathic pulmonary fibrosis. Cough 2010; 6: 4.

25 Nathan SD, Shlobin OA, Weir N, et al. Long-term course and prognosis of idiopathic pulmonary fibrosis in the new millennium. Chest 2011; 140: 221-229.

26 du Bois RM, Weycker D, Albera C, et al. Forced vital capacity in patients with idiopathic pulmonary fibrosis: test properties and minimal clinically important difference. Am J Respir Crit Care Med 2011; 184: 1382-1389.

27 American Thoracic Society and European Respiratory Society. Idiopathic pulmonary fibrosis: diagnosis and treatment. International consensus statement. Am J Respir Crit Care Med 2000; 161: 646-664.

28 Richeldi L, Ryerson CJ, Lee JS, et al. Relative versus absolute change in forced vital capacity in idiopathic pulmonary fibrosis. Thorax 2012; 67: 407-411.

29 Zappala CJ, Latsi PI, Nicholson AG, et al. Marginal decline in forced vital capacity is associated with a poor outcome in idiopathic pulmonary fibrosis. Eur Respir J 2010; 35: 830-835.

30 Russell AM, Adamali H, Molyneaux PL, et al. Daily home spirometry: an effective tool for detecting progression in idiopathic pulmonary fibrosis. Am J Respir Crit Care Med 2016; 194: 989-997.

31 Richeldi L, du Bois RM, Raghu G, et al. Efficacy and safety of nintedanib in idiopathic pulmonary fibrosis. $N$ Engl J Med 2014; 370: 2071-2082.

32 Wuyts WA, Kolb M, Stowasser S, et al. First data on efficacy and safety of nintedanib in patients with idiopathic pulmonary fibrosis and forced vital capacity of $\leqslant 50 \%$ of predicted value. Lung 2016; 194: 739-743.

33 Zisman DA, Schwarz M, Anstrom KJ, et al. A controlled trial of sildenafil in advanced idiopathic pulmonary fibrosis. N Engl J Med 2010; 363: 620-628.

34 King TE, Albera C, Bradford WZ, et al. All-cause mortality rate in patients with idiopathic pulmonary fibrosis: implications for the design and execution of clinical trials. Am J Respir Crit Care Med 2014; 189: 825-831.

35 Nathan SD, Albera C, Bradford WZ, et al. Effect of pirfenidone on mortality: pooled analyses and meta-analyses of clinical trials in idiopathic pulmonary fibrosis. Lancet Respir Med 2016; 5: 33-41.

36 Nathan SD, Albera C, Bradford WZ, et al. Effect of continued treatment with pirfenidone following clinically meaningful declines in forced vital capacity: analysis of data from three phase 3 trials in patients with idiopathic pulmonary fibrosis. Thorax 2016; 71: 429-435.

37 Kolb M, Richeldi L, Behr J, et al. Nintedanib in patients with idiopathic pulmonary fibrosis and preserved lung volume. Thorax 2017; 72: 340-346.

38 Albera C, Costabel U, Fagan EA, et al. Efficacy of pirfenidone in patients with idiopathic pulmonary fibrosis with more preserved lung function. Eur Respir J 2016; 48: 843-851.

39 Sverzellati N, Guerci L, Randi G, et al. Interstitial lung diseases in a lung cancer screening trial. Eur Respir J 2011; 38: 392-400.

40 Wells AU, Kokosi MA. Subclinical interstitial lung abnormalities: toward the early detection of idiopathic pulmonary fibrosis? Am J Respir Crit Care Med 2016; 194: 1445-1446.

41 King TE, Safrin S, Starko KM, et al. Analyses of efficacy end points in a controlled trial of interferon- $\gamma 1 \mathrm{~b}$ for idiopathic pulmonary fibrosis. Chest 2005; 127: 171-177.

42 Latsi PI, du Bois RM, Nicholson AG, et al. Fibrotic idiopathic interstitial pneumonia: the prognostic value of longitudinal functional trends. Am J Respir Crit Care Med 2003; 168: 531-537.

43 Taylor Gonzalez A, Maher T. Predicting mortality in idiopathic pulmonary fibrosis. Which parameters should be used to determine eligibility for treatment? Analysis of a UK prospective cohort. Eur Respir J 2016; 48: suppl 60, $\mathrm{OA} 282$.

44 Corte TJ, Wort SJ, MacDonald PS, et al. Pulmonary function vascular index predicts prognosis in idiopathic interstitial pneumonia. Respirology 2012; 17: 674-680.

45 Pellegrino R, Viegi G, Brusasco V, et al. Interpretative strategies for lung function tests. Eur Respir J 2005; 26: 948-968.

46 Goldin JG, Hansell DM, Goldin JG, et al. CT staging and monitoring of fibrotic interstitial lung diseases in clinical practice and treatment trials: a position paper from the Fleischner Society. Lancet Respir 2015; 3: 483-496.

47 Du Bois RM, Albera C, Bradford WZ, et al. 6-Minute walk distance is an independent predictor of mortality in patients with idiopathic pulmonary fibrosis. Eur Respir J 2014; 43: 1421-1429.

48 Nathan SD, Du Bois RM, Albera C, et al. Validation of test performance characteristics and minimal clinically important difference of the 6-minute walk test in patients with idiopathic pulmonary fibrosis. Respir Med 2015; 109: 914-922.

49 Du Bois RM, Weycker D, Albera C, et al. Six-minute-walk test in idiopathic pulmonary fibrosis: test validation and minimal clinically important difference. Am J Respir Crit Care Med 2011; 183: 1231-1237. 
50 Eaton T, Young P, Milne D, et al. Six-minute walk, maximal exercise tests: reproducibility in fibrotic interstitial pneumonia. Am J Respir Crit Care Med 2005; 171: 1150-1157.

51 Flaherty KR, Andrei AC, Murray S, et al. Idiopathic pulmonary fibrosis: prognostic value of changes in physiology and six-minute-walk test. Am J Respir Crit Care Med 2006; 174: 803-809.

52 Swigris JJ, Swick J, Wamboldt FS, et al. Heart rate recovery after 6-min walk test predicts survival in patients with idiopathic pulmonary fibrosis. Chest 2009; 136: 841-848.

53 Swigris JJ, Olson AL, Shlobin OA, et al. Heart rate recovery after six-minute walk test predicts pulmonary hypertension in patients with idiopathic pulmonary fibrosis. Respirology 2011; 16: 439-445.

54 Ley B, Bradford WZ, Vittinghoff E, et al. Predictors of mortality poorly predict common measures of disease progression in idiopathic pulmonary fibrosis. Am J Respir Crit Care Med 2016; 194: 711-718.

55 Hansell DM, Bankier AA, MacMahon H, et al. Fleischner Society: glossary of terms for thoracic imaging. Radiology 2008; 246: 697-722.

56 Wells AU, Rubens MB, du Bois RM, et al. Serial CT in fibrosing alveolitis: prognostic significance of the initial pattern. Am J Roentgenol 1993; 161: 1159-1165.

57 Wells AU, Hansell DM, Rubens MB, et al. The predictive value of appearances on thin-section computed tomography in fibrosing alveolitis. Am Rev Respir Dis 1993; 148: 1076-1082.

58 Lynch DA, Godwin JD, Safrin S, et al. High-resolution computed tomography in idiopathic pulmonary fibrosis. Am J Respir Crit Care Med 2005; 172: 488-493.

59 Flaherty KR, Thwaite EL, Kazerooni EA, et al. Radiological versus histological diagnosis in UIP and NSIP: surviva implications. Thorax 2003; 58: 143-148.

60 Jeong YJ, Lee KS, Müller NL, et al. Usual interstitial pneumonia and non-specific interstitial pneumonia: serial thin-section CT findings correlated with pulmonary function. Korean J Radiol 2005; 6: 143-152.

61 Edey AJ, Devaraj AA, Barker RP, et al. Fibrotic idiopathic interstitial pneumonias: HRCT findings that predict mortality. Eur Radiol 2011; 21: 1586-1593.

62 Yamauchi H, Bando M, Baba T, et al. Clinical course and changes in high-resolution computed tomography findings in patients with idiopathic pulmonary fibrosis without honeycombing. PLoS ONE 2016; 11: e0166168.

63 Raghu G, Wells AU, Nicholson AG, et al. Effect of nintedanib in subgroups of idiopathic pulmonary fibrosis by diagnostic criteria. Am J Respir Crit Care Med 2016; 195: 1-34.

64 Shin KM, Lee KS, Chung MP, et al. Prognostic determinants among clinical, thin-section CT, and histopathologic findings for fibrotic idiopathic interstitial pneumonias: tertiary hospital study. Radiology 2008; 249: 328-337.

65 Romei C, Tavanti L, Sbragia P, et al. Idiopathic interstitial pneumonias: do HRCT criteria established by ATS/ ERS/JRS/ALAT in 2011 predict disease progression and prognosis? Radiol Medica 2015; 120: 930-940.

66 Sumikawa H, Johkoh T, Colby TV, et al. Computed tomography findings in pathological usual interstitial pneumonia. Am J Respir Crit Care Med 2008; 177: 433-439.

67 Lee HY, Lee KS, Jeong YJ, et al. High-resolution CT findings in fibrotic idiopathic interstitial pneumonias with little honeycombing: serial changes and prognostic implications. Am J Roentgenol 2012; 199: $982-989$.

68 Hwang J-H, Misumi S, Curran-Everett D, et al. Longitudinal follow-up of fibrosing interstitial pneumonia: relationship between physiologic testing, computed tomography changes, and survival rate. J Thorac Imaging 2011; 26: 209-217.

69 Oda K, Ishimoto H, Yatera K, et al. High-resolution CT scoring system-based grading scale predicts the clinical outcomes in patients with idiopathic pulmonary fibrosis. Respir Res 2014; 15: 10.

70 Hartley PG, Galvin JR, Hunninghake GW, et al. High-resolution CT-derived measures of lung density are valid indexes of interstitial lung disease. J Appl Physiol 1994; 76: 271-277.

71 Best AC, Lynch AM, Bozic CM, et al. Quantitative CT indexes in idiopathic pulmonary fibrosis: relationship with physiologic impairment. Radiology 2003; 228: 407-414.

72 Sverzellati N, Calabrò E, Chetta A, et al. Visual score and quantitative CT indices in pulmonary fibrosis: relationship with physiologic impairment. Radiol Medica 2007; 112: 1160-1172.

73 Maldonado F, Moua T, Rajagopalan S, et al. Automated quantification of radiological patterns predicts survival in idiopathic pulmonary fibrosis. Eur Respir J 2014; 43: 204-212.

74 Jacob J, Bartholmai BJ, Rajagopalan S, et al. Automated quantitative computed tomography versus visual computed tomography scoring in idiopathic pulmonary fibrosis: validation against pulmonary function. $J$ Thorac Imaging 2016; 31: 304-311.

75 Jacob J, Bartholmai BJ, Rajagopalan S, et al. Mortality prediction in idiopathic pulmonary fibrosis: evaluation of automated computer tomographic analysis with conventional severity measures. Eur Respir J 2017; 49: pii:1601011.

76 Salisbury ML, Lynch DA, van Beek EJR, et al. Idiopathic pulmonary fibrosis: the association between the adaptive multiple features method and fibrosis outcomes. Am J Respir Crit Care Med 2017; 195: 921-929.

77 Watadani T, Sakai F, Johkoh T, et al. Interobserver variability in the CT assessment of honeycombing in the lungs. Radiology 2013; 266: 936-944

78 Nusair S, Rubinstein R, Freedman NM, et al. Positron emission tomography in interstitial lung disease. Respirology 2007; 12: 843-847.

79 Groves AM, Win T, Screaton NJ, et al. Idiopathic pulmonary fibrosis and diffuse parenchymal lung disease: implications from initial experience with 18F-FDG PET/CT. J Nucl Med 2009; 50: 538-545.

80 Meissner H-H, Soo Hoo GW, Khonsary SA, et al. Idiopathic pulmonary fibrosis: evaluation with positron emission tomography. Respiration 2006; 73: 197-202.

81 Umeda Y, Demura Y, Ishizaki T, et al. Dual-time-point ${ }^{18}$ F-FDG PET imaging for diagnosis of disease type and disease activity in patients with idiopathic interstitial pneumonia. Eur J Nucl Med Mol Imaging 2009; 36: $1121-1130$.

82 Umeda Y, Demura Y, Morikawa M, et al. Prognostic value of dual-time-point ${ }^{18}$ F-FDG PET for idiopathic pulmonary fibrosis. J Nucl Med 2015; 56: 1869-1875.

83 King MA, Bergin CJ, Ghadishah E, et al. Detecting pulmonary abnormalities on magnetic resonance images in patients with usual interstitial pneumonitis: effect of varying window settings and gadopentetate dimeglumine. Acad Radiol 1996; 3: 300-307. 
84 Rea G. Magnetic resonance imaging in the evaluation of idiopathic pulmonary fibrosis: a real possibility, or an attractive challenge? Quant Imaging Med Surg 2016; 6: 331-333.

85 Yi CA, Lee KS, Han J, et al. 3-T MRI for differentiating inflammation- and fibrosis-predominant lesions of usual and nonspecific interstitial pneumonia: comparison study with pathologic correlation. Am J Roentgenol 2008; 190: 878-885.

86 Lavelle LP, Brady D, McEvoy S, et al. Pulmonary fibrosis: tissue characterization using late-enhanced MRI compared with unenhanced anatomic high-resolution CT. Diagnostic Interv Radiol 2017; 23: 106-111.

87 Mirsadraee S, Tse M, Kershaw L, et al. T1 characteristics of interstitial pulmonary fibrosis on 3T MRI-a predictor of early interstitial change? Quant Imaging Med Surg 2016; 6: 42-49.

88 Ley B, Ryerson CJ, Vittinghoff E, et al. A multidimensional index and staging system for idiopathic pulmonary fibrosis. Ann Intern Med 2012; 156: 684-695.

89 Daccord C, Maher TM. Recent advances in understanding idiopathic pulmonary fibrosis. F1000 Res 2016; 5: 1-13.

90 Ley B, Bradford WZ, Weycker D, et al. Unified baseline and longitudinal mortality prediction in idiopathic pulmonary fibrosis. Eur Respir J 2015; 45: 1374-1381.

91 Salisbury ML, Xia M, Zhou Y, et al. Idiopathic pulmonary fibrosis: gender-age-physiology index stage for predicting future lung function decline. Chest 2016; 149: 491-498.

92 Kolb M, Collard HR. Staging of idiopathic pulmonary fibrosis: past, present and future. Eur Respir Rev 2014; 23: 220-224.

93 Flaherty KR, Travis WD, Colby TV, et al. Histopathologic variability in usual and nonspecific interstitial pneumonias. Am J Respir Crit Care Med 2001; 164: 1722-1727.

94 Wolters P, Collard H, Jones K. Pathogenesis of idiopathic pulmonary fibrosis. Annu Rev Pathol 2014; 9: 157-179.

95 Nicholson AG, Fulford LG, Colby TV, et al. The relationship between individual histologic features and disease progression in idiopathic pulmonary fibrosis. Am J Respir Crit Care Med 2002; 166: 173-177.

96 Walsh SLF, Wells AU, Sverzellati N, et al. Relationship between fibroblastic foci profusion and high resolution CT morphology in fibrotic lung disease. BMC Med 2015; 13: 241.

97 Wells AU. "Any fool can make a rule and any fool will mind it". BMC Med 2016; 14: 23.

98 Nicholson AG, Addis BJ, Bharucha $\mathrm{H}$, et al. Inter-observer variation between pathologists in diffuse parenchymal lung disease. Thorax 2004; 59: 500-505. 